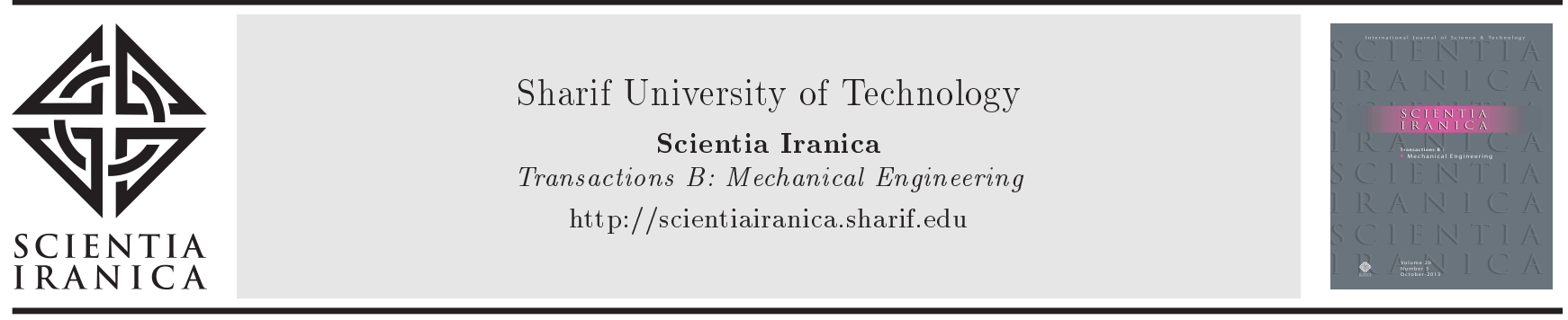

\title{
Hypersonic flow over hemispherical blunt body with spikes
}

\author{
S. Das ${ }^{a, *}$, P. Kumar ${ }^{a}$, and J.K. Prasad ${ }^{b}$ \\ a. Department of Space Engineering and Rocketry, Birla Institute of Technology, Mesra, Ranchi, JH-835215, India. \\ b. Birla Institute of Technology, Deoghar, Jharkhand, India.
}

Received 23 May 2017; received in revised form 27 July 2017; accepted 23 April 2018

\author{
KEYWORDS \\ Hemispherical nose \\ cone; \\ Drag reduction; \\ Hemispherical head \\ spike; \\ Recirculation zone; \\ Flow separation.
}

\begin{abstract}
Use of spike on a hemispherical body changes the flow field and hence, the aerodynamic drag. Computational studies have been carried out to obtain the flow field around a hemispherical body with spikes at a hypersonic Mach number of 6 . The effect of shape of spike tip and length has been studied. Laminar computations have been made adopting structured grid using commercial software FLUENT. It is observed that use of a sharp spike itself reduces the drag significantly. However, the use of a hemispherical head spike further reduces the drag. Contribution of different components towards drag indicates that the increase in length of a spike does not change the spike contribution. However, the flow field on main body is altered, which leads to reduction in drag with change in length. The estimated maximum drag is found to be highest among all reported drag values with any spike shape and length in the literature.
\end{abstract}

(C) 2019 Sharif University of Technology. All rights reserved.

\section{Introduction}

The majority of nose shapes of aerospace structures, such as missiles, rockets, reentry vehicles, etc., are blunt in nature, due to several considerations. Highspeed hypersonic flows over such blunt shaped bodies generate a bow shock wave ahead of the body, which leads to high wave drag and heating rates. The consequence could be a poor performance and attrition of body surface. Various methods have been adopted in the past to minimize the adverse effects of the drag and high heating rates. Use of a spike at the tip of blunt body is the simplest and an efficient technique among other methods such as counterjet flow, energy deposition, breathing nose, etc. A schematic showing hypersonic flow over a typical hemispherical blunt body

*. Corresponding author. Tel.: +919431178604 E-mail addresses: sudipdas@bitmesra.ac.in (S. Das); priyankkumar@bitmesra.ac.in (P. Kumar)

doi: $10.24200 /$ sci.2018.20339 without and with a sharp spike is shown in Figure 1. Presence of spike generates a weaker tip shock, leading to formation of a separation zone on the spike stem due to adverse pressure gradient, recirculation zone, formation of shear layer separating the main flow and recirculation zone, reattachment shock on the main body, etc. These flow features depend on freestream Mach number, Reynolds number, spike shape, length and diameter of spike stem, etc. The flow ahead of main body gets altered due to the presence of spike. Significant drop in pressure is expected on the leading edge of the main body and adjacent surface, which could lead to reduction in the drag and heating values.

Systematic experiments by Crawford [1] on a hemispherically blunt body at $\mathrm{M}=6.8$ with several lengths of sharp spikes were reported, among the few early research works on such concepts. A drag reduction of the order $69 \%$ was reported in this study. Similar experiments were also reported by Maull [2] emphasizing the unsteady flows due to spike. Numerical simulations with emphasis on capturing unsteady flows on cone frustum with spike were reported by 


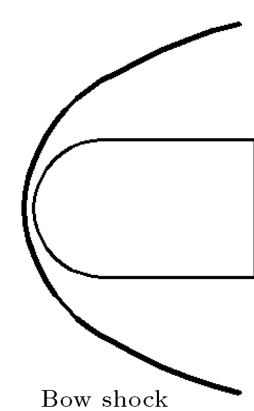

(a) Without spike

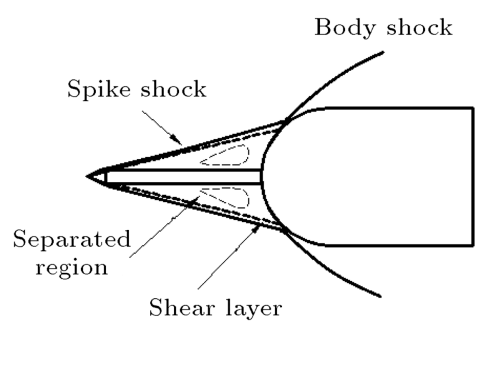

(b) With spike
Figure 1. Schematic of flow field around a blunt body without and with a spike.

Panaras and Drikakis [3] at $\mathrm{M}=6.0$. Menezes et al. [4] tested the effectiveness of spikes on blunt faced cones at $\mathrm{M}=5.75$, and could achieve a drag reduction of the order $65 \%$ with a flat faced aerodisc spike. Mach 6.0 experiments with variations in geometrical parameters of spike and different shapes were tested by Kalimuthu et al. [5], who reported that the aerodisc configuration performed better than aerospikes. However, in the same line, Kalimuthu and Rathakrishnan [6] reported that with a hemispherical aerospike at $L / D=2.0$, there was a drag reduction of about $78 \%$.

The mechanism of drag reduction with spikes of different diameters and lengths at $\mathrm{M}=6.0$ using extensive computations was reported by Ahmed and Qin [7-9]. Numerical studies made by Gauer and Paull [10] at Mach numbers from 5.0 to 10.0 with various $L / D$ 's of spikes (1.0 to 4.0 ) indicated maximum drag reduction of $30 \%$ with sharp spike, $50 \%$ with blunt spike, and $62 \%$ with aerodome spike. Computation at $\mathrm{M}=6.0$ with conical, hemispherical, and a flat faced aerodisk spikes achieved a maximum drag reduction of $60 \%$ in the reports by Tahani et al. [11]. Gerdroodbary and Hosseinalipour [12] reported computations at $\mathrm{M}=5.75$ for a highly blunted cone and spikes of different shapes. Hemispherically blunt spike exhibited maximum drag reduction of $73 \%$. A drag reduction of $77 \%$ was reported by Humieres and Stollery [13] for a reentry capsule shape with sharp spike at $\mathrm{M}=$ 8.2. Quantification of pressures and their reduction with spiked blunt body at $\mathrm{M}=6.0$ was reported by Sebastian et al. [14]. Drag reduction of the order 86 to $90 \%$ was reported at $\mathrm{M}=6.0$ by Eghlima and Mansour [15] through computations, adopting a combination of spike and counterflow jet. Thermal analysis of spikes at $\mathrm{M}=5.0$ with different shapes and diameters was carried out by Qin et al. [16]. Numerical simulations with series of aerospikes at $\mathrm{M}=10.0$ were performed by Yadav et al. [17], which reported a maximum drag reduction of $44 \%$.

It is observed that many combinations of spike shape, diameter, length to diameter, etc. have already been studied, which enhance the drag reduction capability up to $78 \%$. It is also clear that only the combination of two drag reducing methods could produce a drag reduction up to $90 \%$. In the present investigation, attempt is made to maximize the drag reduction by using a suitable spike at $\mathrm{M}=6.0$ using computations. Our recent experimental and computational work [18] at supersonic Mach number on several spikes of different shapes has led to a drag reduction of around $46 \%$. However, the present work is an extension of the work being carried out at supersonic Mach number [19] with a typical spike of hemispherical tip with an aft faring flare. This typical spike experimentally exhibited a drag reduction of $68 \%$. The thrust of the present work is anticipation of a better drag reducing capability of this spike at hypersonic speed.

\section{Geometry}

The geometry adopted in the present study is a hemispherical blunt body of diameter $D$ and length $1.5 D$. Two different spike tip shapes (sharp and blunt) are used with fixed spike stem diameter of $0.1 D$. The sharp spike has a tip with semi cone angle of 20 degrees, while the blunt spike has a hemispherical tip with a diameter of $0.2 D$ and a flare angle of 135 degrees, as shown in Figure 2. Three different spike lengths $(L / D=0.75,1.0,1.5)$ are used for both of the spikes. The nomenclature of the spikes used is " $X X-Y Y Y$ ", where $X X$ denotes the shape of the spike and, in the present case, it is either sharp (SS) or hemispherical (HS) and $Y Y Y$ represents length of spike $(L / D)$, which has the value of $0.75,1.00$, or 1.50 .

\section{Computation}

To obtain the flow features of blunt body with and

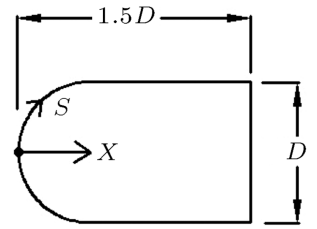

(a) Hemispherical body

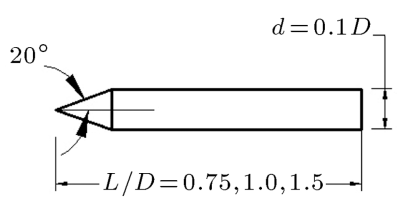

(b) Sharp Spike (SS)

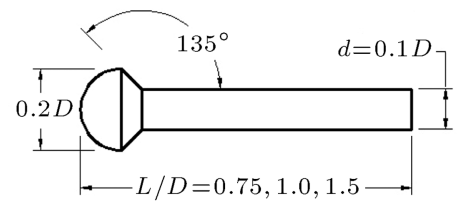

(c) Hemispherical Head Spike (HS)

Figure 2. Geometrical details of models. 


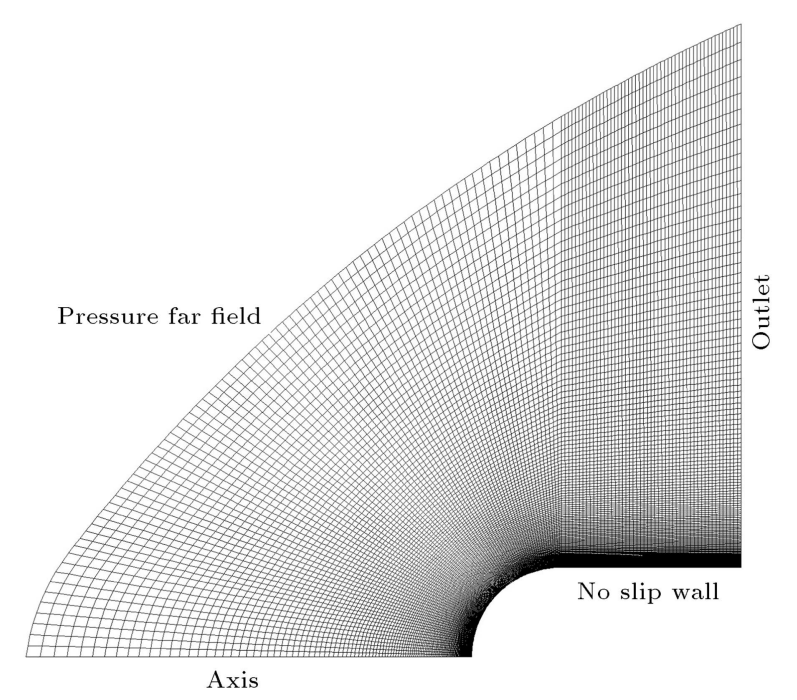

Figure 3. Computational domain and boundary conditions.

Table 1. Operating conditions for present simulation.

\begin{tabular}{cl}
\hline Flow parameter & \multicolumn{1}{c}{ Value } \\
\hline$M_{\infty}$ & 6.0 \\
$T_{\infty}$ & $221.6 \mathrm{~K}$ \\
$P_{\infty}$ & $2511 \mathrm{~Pa}$ \\
$\operatorname{Re}_{D}$ & $0.73 \times 10^{5}$ \\
$\rho_{\infty}$ & $0.03947 \mathrm{~kg} / \mathrm{m}^{3}$ \\
$U_{\infty}$ & $1790 \mathrm{~m} / \mathrm{s}$ \\
\hline
\end{tabular}

without spikes, numerical simulations were made using FLUENT, which used a finite volume approach to solve compressible Reynolds Averaged Navier Stokes equations. In the present investigation, steady state laminar axisymmetric computations were made adopting explicit coupled solver. Structured grids were made with uniformly distributed quadrilateral cells having minimum spacing near the wall of the order $10^{-2} \mathrm{~mm}$. The overall computational domain was obtained after several iterations. Pressure far field boundary condition was specified at the inlet. No slip wall boundary conditions were enforced on the hemispherical body and spike. Due to axisymmetry, only half domain with axis boundary condition was applied at the centerline. The overall grid, computational domain, and boundary conditions adopted are shown in Figure 3. The operating conditions applied in the present simulation are listed in Table 1. Close view of typical grids used in the present investigation for different configurations is presented in Figure 4. Necessary clustering of grids was made to better capture of flow field. Final grid was obtained after making necessary grid sensitivity tests, and performing proper comparison with the experimental and numerical results reported in the literature.

The residuals of continuity and energy along

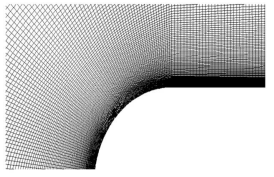

(a) Without spike

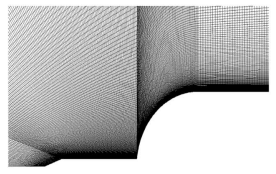

(b) $\mathrm{SS}-0.75$

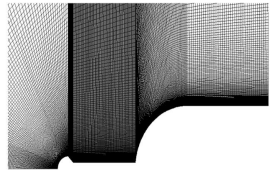

(c) HS-0.75
Figure 4. Grids for hemispherical models without and with different spikes.

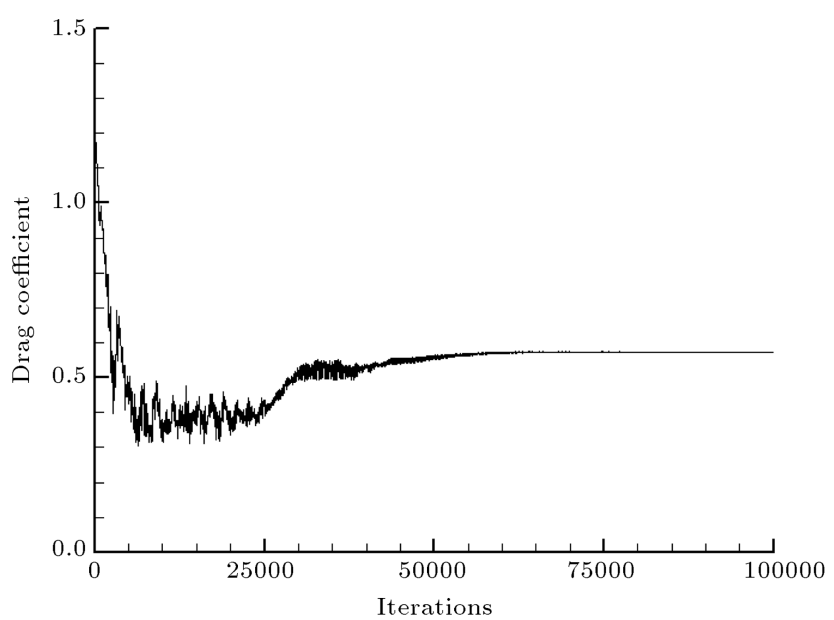

Figure 5. Convergence history of drag for SS-0.75.

with mass flux between the inflow and outflow were monitored. In addition, the convergence history for drag was monitored during the entire solution period. Results were analyzed only when it was ascertained that the residuals had converged on the order of $10^{-3}$. A typical convergence history of drag for hemispherical body with sharp spike is plotted in Figure 5 .

\section{Validation}

To validate the present computations and to arrive at a suitable grid as well, experimental results reported in [1] have been used. One of the experimental cases involves a hemispherical body with a sharp spike having $L / D=2.0$ at freestream condition of $\mathrm{M}=6.8$, $\operatorname{Re}_{D}=0.15 \times 10^{6}$. Similar conditions and geometry were simulated using three different resolutions of grids [coarse $(26,250)$, medium $(52,500)$, and fine $(105,000)$ ] and the results obtained are presented in Figure 6 and Table 2. Figure shows the similarity of pressures on the hemispherical body for medium grid with the results reported in the literature. It is also observed that the difference between the values of $C_{d f}$ for coarse to medium is of the order 0.19 , while for medium to fine grid, it is only 0.05 . Comparison with the reported value of $C_{d}$ shows good agreement for medium grid. Hence, further computations are made with medium grids having cells of the order 52,500. Simulations are also made for sharp spike having $L / D=1.0$ and 2.0 to compare the reported Schlieren and surface pressures 


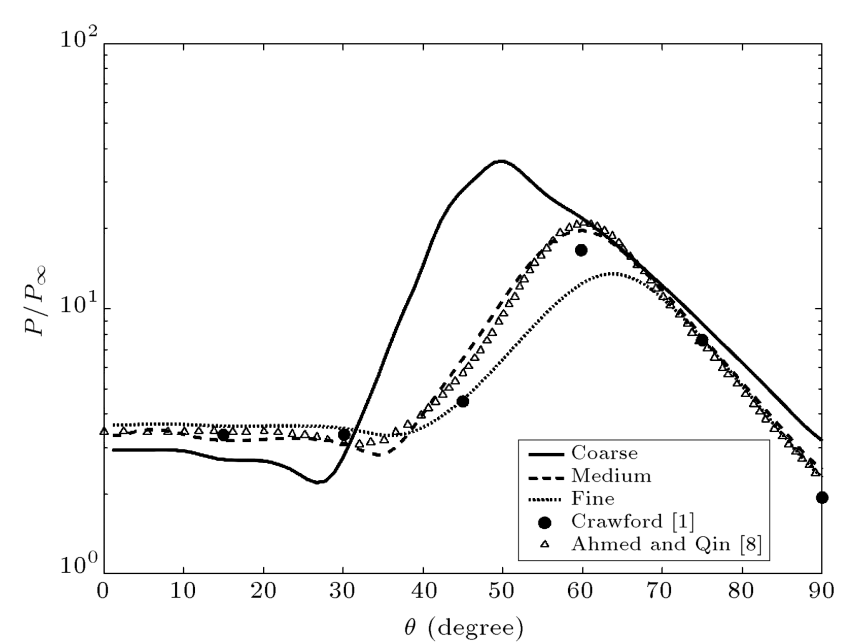

Figure 6. Comparison of pressure distribution for the tested grids.

on the blunt body. Comparison of numerical and experimental Schlieren, presented in Figure 7, indicates that the overall flow features are well captured. The comparison of surface pressure distributions on blunt body without spike and with sharp spike having the lengths $L / D=1.0$ and 2.0 is presented in Figure 8, which indicates fairly good agreement. The comparison of forebody drag with the reported values for sharp spike with various $L / D$ is presented in Figure 9, which also indicates good agreement. These results indicate the adequacy of the grids, domain, and boundary

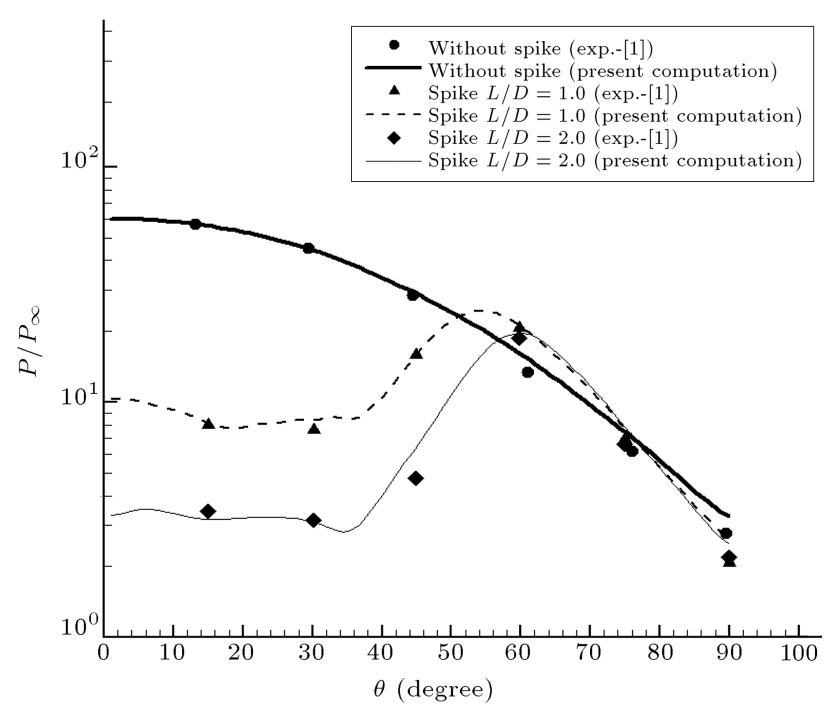

Figure 8. Comparison of pressure distribution at Mach number of 6.8 .

conditions and, hence, similar type of conditions has been adopted in the present investigation.

\section{Results}

Computations have been made to investigate the overall flow field over hemispherical body with and without spike at Mach 6.0. The density gradient contours obtained for hemispherical body without spike and

Table 2. Grid sensitivity test for hemispherical body with a sharp spike and $L / D=2.0$.

\begin{tabular}{cclcc}
\hline Grid & Number of cells & $\begin{array}{c}\text { Near wall } \\
\text { cell height }\end{array}$ & $\boldsymbol{C}_{\boldsymbol{d f}}$ & $\begin{array}{c}\text { Difference from } \\
\text { experiments [1] }\end{array}$ \\
\hline Coarse & 26,250 & $6.5 \times 10^{-3} D$ & 0.41 & 0.2 \\
Medium & 52,500 & $10^{-3} D$ & 0.22 & 0.01 \\
Fine & 105,000 & $6.5 \times 10^{-4} D$ & 0.17 & 0.04 \\
Ahmed and Qin [8] & $3,50,000$ & $10^{-4} D$ & 0.21 & 0 \\
Crawford [1] & Experiments & & 0.21 & - \\
\hline
\end{tabular}

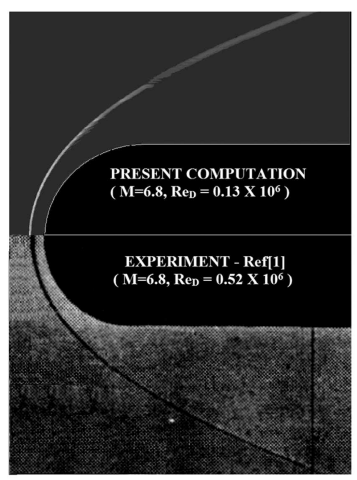

(a) Without spike

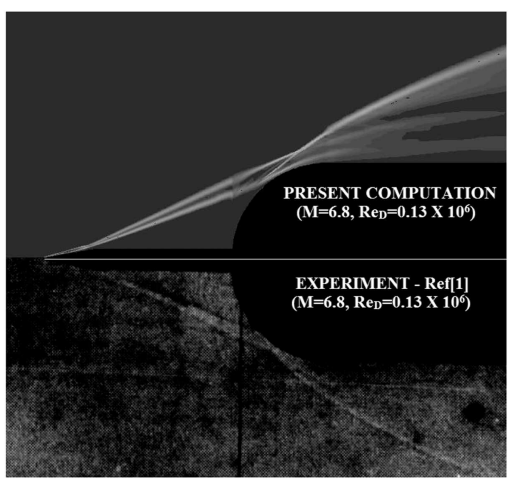

(b) Sharp spike $(L / D=1.0)$

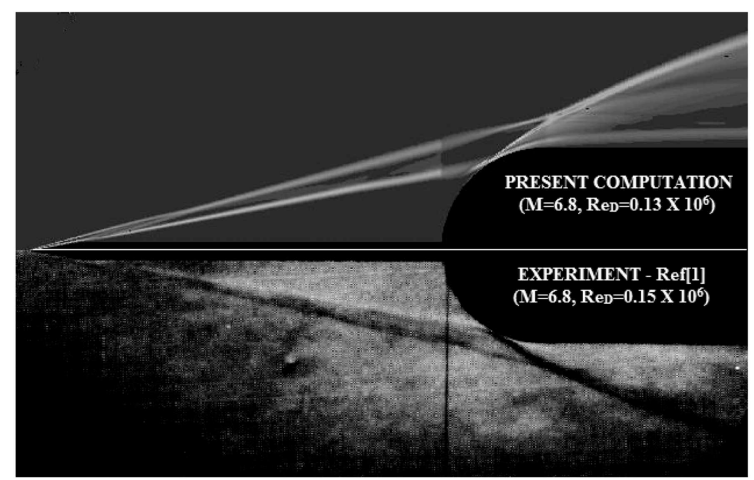

(c) Sharp spike $(L / D=2.0)$

Figure 7. Comparison of present computation with Schlieren for hemispherical body without spike and with different lengths of sharp spike. 


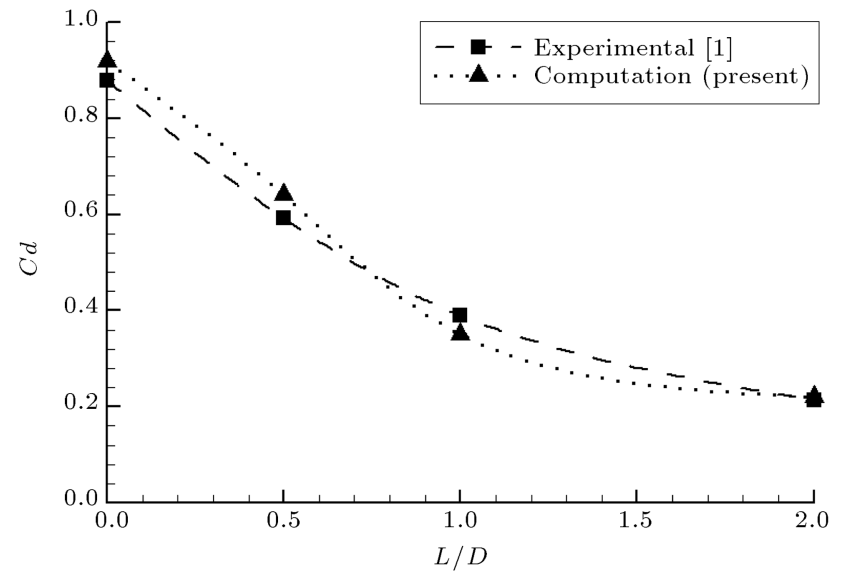

Figure 9. Comparison of drag for different $L / D$ 's of spike.

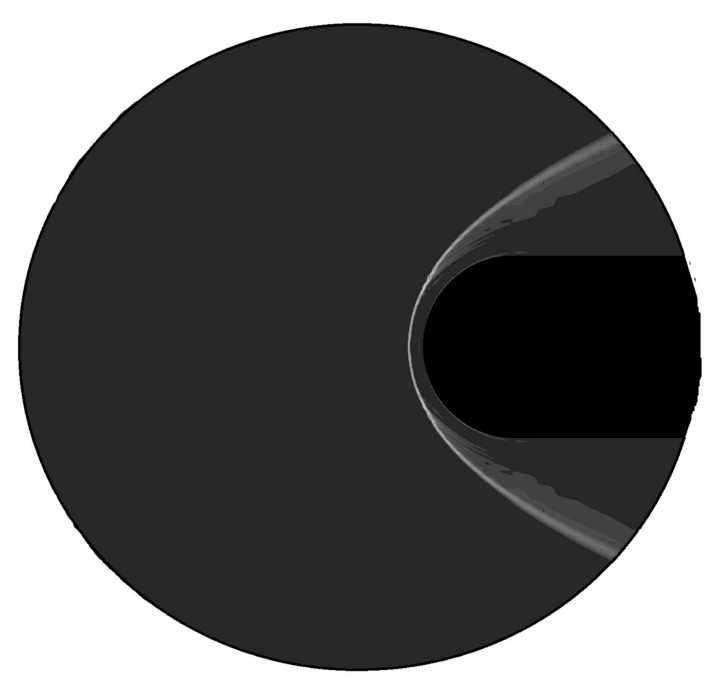

Figure 10. Density gradient contours over hemispherical body without spike.

with different spikes through computation are shown in Figures 10 and 11, respectively. The flow field around hemispherical body without spike indicates a strong detached bow shock wave in front of the hemispherical body, which generates strong drag and heating load. With adoption of sharp spike of $L / D=0.75$ (SS0.75 ), a conical shock is observed, which emanates from the spike tip (Figure 11(a)). In addition, it is observed that a shear layer exists from the spike cone cylinder junction to the blunt body, which could be due to separation zone over the spike stem. The shear layer envelops the recirculation region near the spike stem. The body shock, which is the strong detached bow shock, now gets altered and nearly attaches to the surface of the blunt body. With increase in length of spike $(L / D=1.0$ and 1.5$)$, the distance between the spike shock and the shear layer increases (Figure 11(b) and (c)). With increase in spike length, the body shock curvature is also observed to move closer to the blunt body. Similar qualitative features are obtained

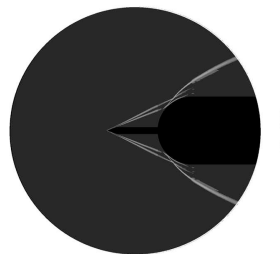

(a) SS-0.75

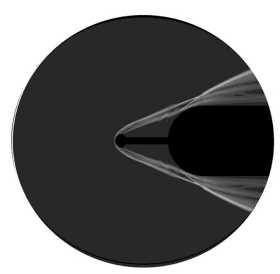

(d) HS-0.75

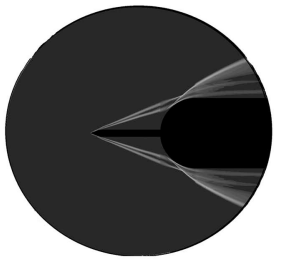

(b) SS-1.00

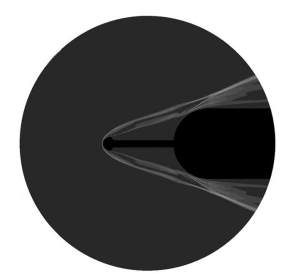

(e) HS-1.00

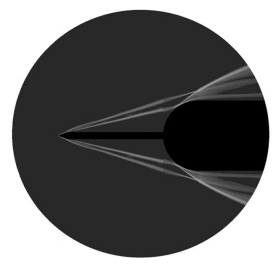

(c) SS-1.50

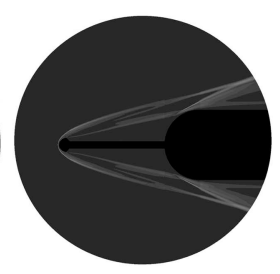

(f) $\mathrm{HS}-1.50$
Figure 11. Density gradient contours over hemispherical body with different shapes and lengths of spike.

with adoption of different lengths of hemispherical head spike (Figure 11(d), (e), and (f)). The observed differences are in the locations of the start of shear layer, spike shocks, and the blunt body shocks. In all the cases of hemispherical head spike, the blunt body shock curvature is more towards the body than towards the sharp spike. Pressure contours presented in Figure 12 show the detailed flow features for all the spikes. The pressures, ahead of the blunt body over the spike and on the blunt body near body shock, decrease with increase in length of the spike for the case of sharp spike of different lengths (Figure 12(a) to (c)). Similar trends are also observed for the hemispherical head spikes shown in Figure 12(d) to (f). In addition, the pressures near the blunt body shock for hemispherical head spike seem to be weaker than those for the sharp spike, probably due to stronger spike tip shock in case of hemispherical head spike. Velocity vectors within the range of Mach numbers from 0 to 1.0, and streamline patterns near the spike and blunt body junction for SS-1.50 and HS-1.50 are presented in Figure 13. The vectors and streamlines are visible only between the spike stem and the shear layer from spike tip to the blunt body surface. It is also clearly visible that the separation zone between the spike stem and the shear layer is larger for the hemispherical head spike than for the sharp spike, which could be responsible for higher reduction in pressure and, hence, drag.

The pressure distribution on the spike and blunt body for sharp spike of different lengths is presented in Figure 14. Blunt body without spike indicates a very high pressure at the tip of the hemispherical body $(S / D=0)$, which is due to the strong bow shock ahead of it; the pressure is subsequently reduced as we move towards the shoulder of the body. With adoption of sharp spike and $L / D=0.75$, the pressure drastically drops to a lower value on the spike and continues up to 


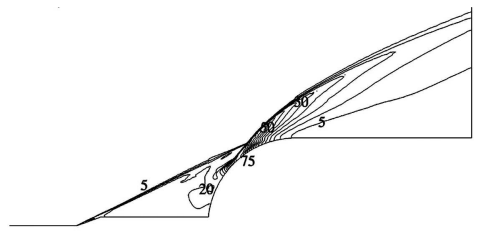

(a) SS-0.75

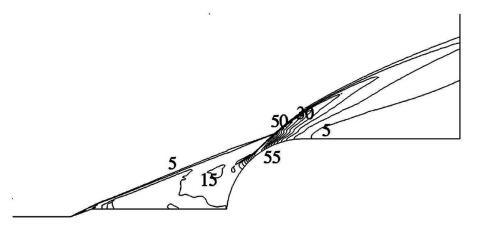

(b) SS-1.00

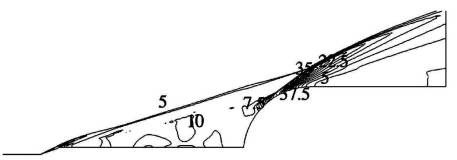

(c) SS-1.50

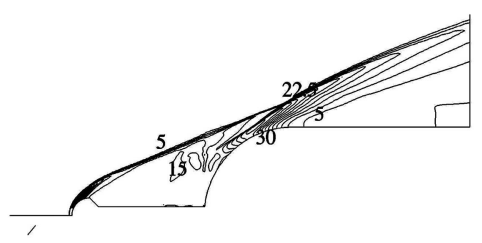

(d) HS- 0.75

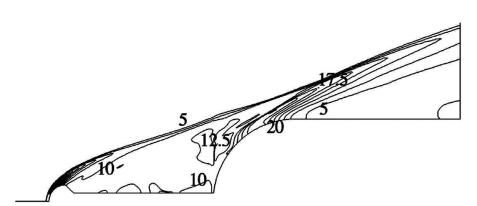

(e) HS-1.00

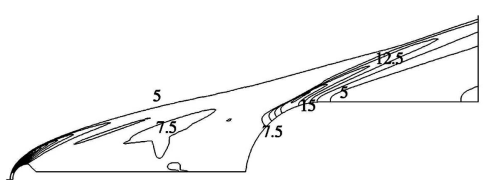

(f) $\mathrm{HS}-1.50$

Figure 12. Pressure $(\mathrm{kPa})$ contours with spikes of different shapes and lengths.

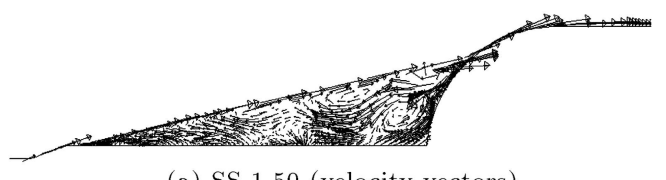

(a) SS-1.50 (velocity vectors)

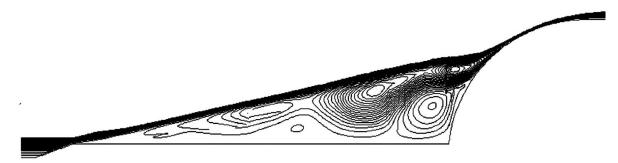

(b) SS-1.50 (streamlines)

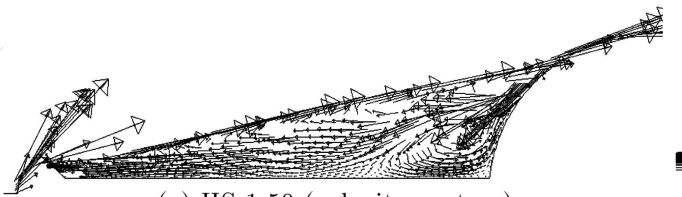

(c) HS-1.50 (velocity vectors)

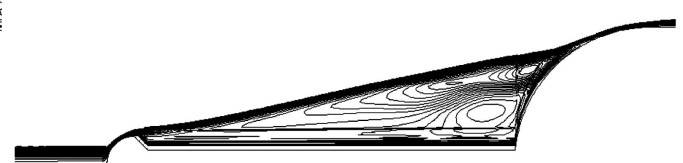

(d) HS-1.50 (streamlines)

Figure 13. Flow patterns over spikes of different shapes and $L / D=1.50$.

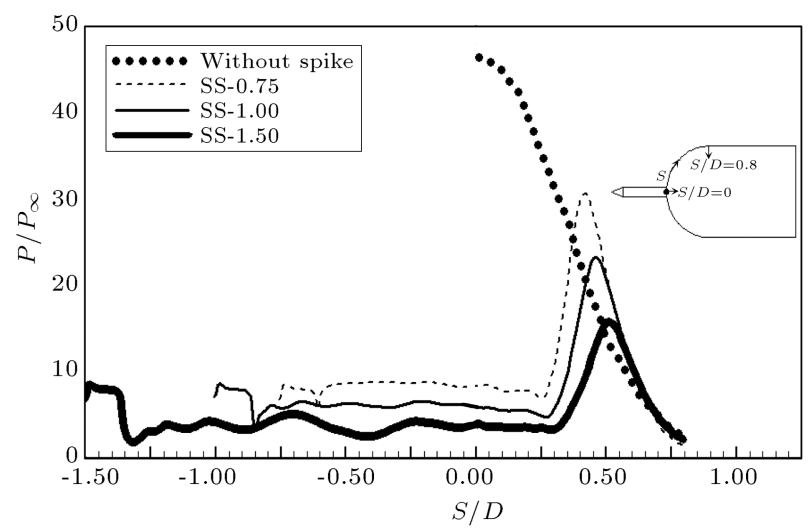

Figure 14. Computed pressure distribution with sharp spike.

$S / D=0.25$; then, it rises on the blunt body to match up with the isolated blunt body pressure. With change in length of the sharp spike, there seems to be a spatial delay in catching up with the blunt body pressure. Maximum pressure on the blunt body and its location change with length of the spike. Figure 15 presents the pressure distribution on the spike and blunt body for hemispherical head spike having different lengths. The maximum peak pressure on the blunt body due

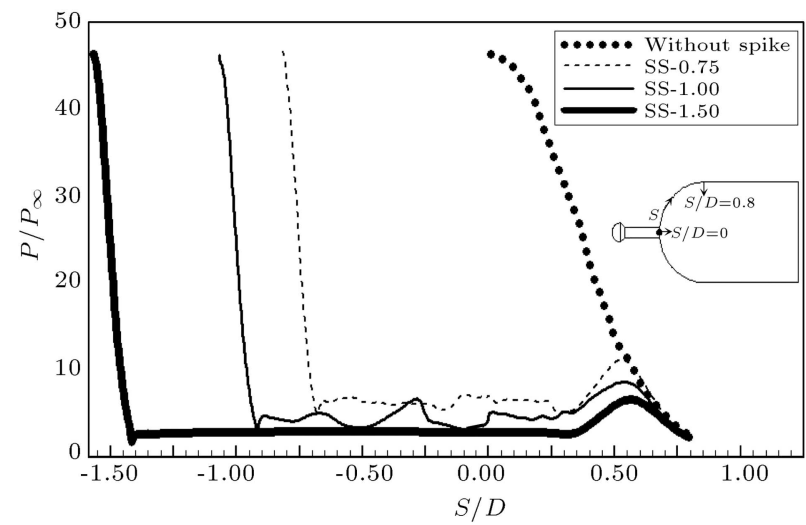

Figure 15. Computed pressure distribution with hemispherical head spike.

to spike drastically drops compared to sharp spike. The length of the spike is also seen to be marginally influencing the peak pressure. This gives a clear indication of lower pressures on the blunt body surface due to hemispherical head spike compared to sharp spike. The estimated values of forebody drag coefficient for the hemispherical body with and without different spikes of various lengths are presented in Figure 16. Isolated hemispherical body shows a drag coefficient 
Table 3. Drag coefficients for sharp and hemispherical head spikes with different lengths.

\begin{tabular}{cccccc}
\hline Configuration & $\boldsymbol{C}_{\boldsymbol{d f}}$ & Reduction (\%) & $\begin{array}{c}\text { Drag component } \\
\text { spike tip }\end{array}$ & $\begin{array}{c}\text { Drag component } \\
\text { spike stem }\end{array}$ & $\begin{array}{c}\text { Drag component } \\
\text { main body }\end{array}$ \\
\hline Without Spike & 0.92 & - & - & - & -0.0000018 \\
SS-0.75 & 0.57 & 38.04 & 0.0035 & 0.00009 & 0.569 \\
SS-1.00 & 0.42 & 54.34 & 0.0035 & 0.00028 & 0.419 \\
SS-1.50 & 0.28 & 69.56 & 0.0035 & -0.00003 & 0.273 \\
HS-0.75 & 0.31 & 66.30 & 0.0381 & -0.00008 & 0.274 \\
HS-1.00 & 0.25 & 72.82 & 0.0382 & -0.00013 & 0.219 \\
HS-1.50 & 0.17 & 81.52 & 0.0383 & 0.136 \\
\hline
\end{tabular}

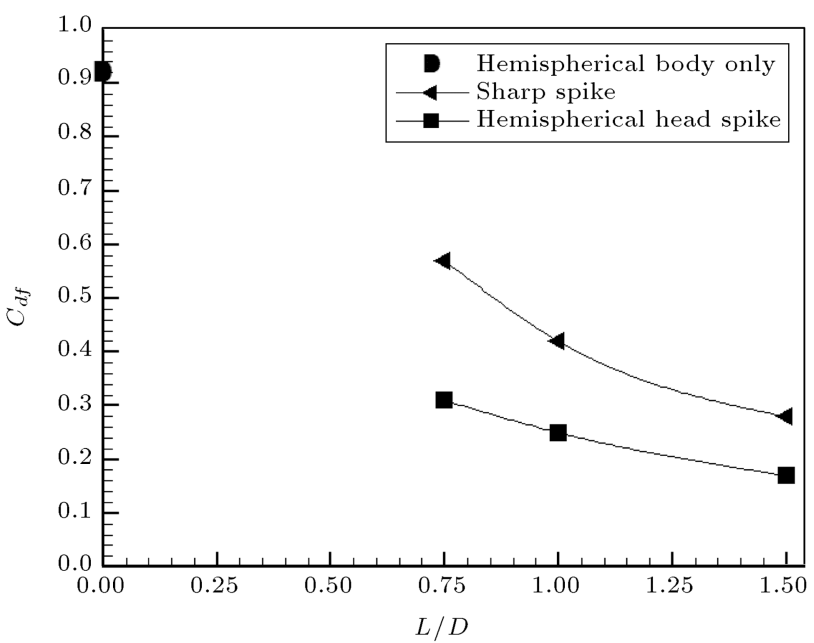

Figure 16. Forebody drag coefficient for different spikes.

of 0.92 , which on adoption of sharp spike indicates a reduction from 0.57 to 0.28 . It is also observed from the figure that with increase in length of the spike, the drag drops linearly. With adoption of the hemispherical head spike, the drag decreases from 0.31 to 0.17 for different lengths of the spike. The details of the drag values for all the cases segregating component-wise and percentage reduction for each case are tabulated in Table 3. This clearly indicates the advantage of the hemispherical head spike, which also shows its advantage in terms of large separating region in velocity vectors and drop in maximum pressure on the blunt body through pressure distribution. Moreover, Figure 16 shows that the spike's $L / D$ influences the reduction in drag and this reduction is also a function of spike tip flow field. The drag reduction percentages for blunt body with sharp and hemispherical head spikes, with various $L / D^{\prime} s$, are presented in Figure 17. Sharp spike exhibits a maximum drag reduction of $69.5 \%$, while the hemispherical head spike has a maximum drag reduction of $81.5 \%$. The drag reduction of $81.5 \%$ is only due to change of the shape of spike tip. This seems to be more than the value of $78 \%$ at hypersonic speed reported in the literature so far.

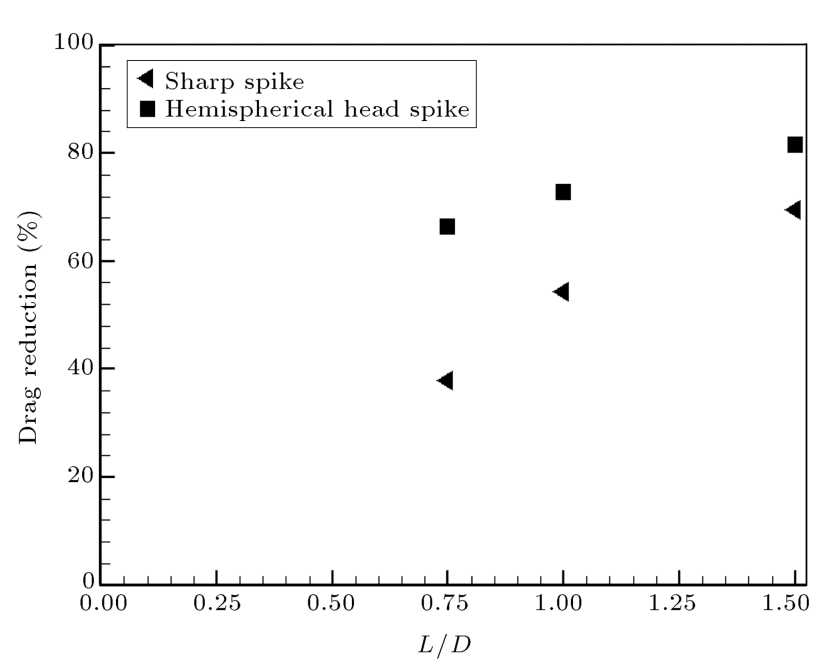

Figure 17. Forebody drag reduction for different spikes.

\section{Conclusion}

Computations were made to obtain the flow field on a hemispherical main body at a hypersonic Mach number of 6 , in the presence of a sharp spike and hemispherical head spike of different lengths. Overall flow features were captured and changes in spike length and shape were obtained. It was observed that use of hemispherical head spike having length of 1.5 times the base diameter led to maximum reduction in drag $(81.5 \%)$. The component-wise drag estimation indicated that the major contribution to the overall drag reduction was only by the main body, which was altered due to spike flow field. Hence, spike tip design was important in the overall drag reduction of the body.

\section{Nomenclature \\ $C_{d f} \quad$ Coefficient of forebody drag \\ $d \quad$ Diameter of stem of spike \\ $D \quad$ Hemispherical body diameter \\ $H S \quad$ Hemispherical head spike \\ $L \quad$ Length of spike}




$\begin{array}{ll}\text { M } & \text { Mach number } \\ P & \text { Pressure }(\mathrm{Pa}) \\ P_{\infty} & \text { Freestream pressure } \\ P_{0} & \text { Total pressure } \\ \mathrm{Re} & \text { Reynolds number } \\ S & \text { Curve length along the surface } \\ & \text { measured from hemispherical body tip } \\ \mathrm{SS} & \text { Sharp Spike } \\ X & \text { Length along the axis of symmetry } \\ & \text { measured from hemispherical body tip }\end{array}$

\section{References}

1. Crawford, D.H., Investigation of Flow Over a Spiked Nose Hemisphere-Cylinder at Mach Number of 6.8, NASA TN D 118 (1959).

2. Maull, D.J. "Hypersonic flow over axially symmetric spiked bodies", Journal of Fluid Mechanics, 8, pp. 584592 (1960)

3. Panaras, A.G. and Drikakis, D. "High-speed unsteady flows around spiked-blunt bodies", Journal of Fluid Mechanics, 632, pp. 69-96 (2009).

4. Menezes, V., Saravanan, S., and Reddy, K.P.J. "Shock tunnel study of spiked aerodynamic bodies flying at hypersonic Mach numbers", Shock Waves, 12, pp.197204 (2002).

5. Kalimuthu, R., Mehta, R.C., and Rathakrishnan, E. "Experimental investigation on spiked body in hypersonic flow", The Aeronautical Journal, 112(1136), pp. 593-598 (2008).

6. Kalimuthu, R. and Rathakrishnan, E. "Aerospike for drag reduction in hypersonic flow", AIAA-2008-4707 (2008).

7. Ahmed, M.Y.M. and Qin, N. "Drag reduction using aerodisks for hypersonic hemispherical bodies", Journal of Spacecraft and Rockets, 47(1), pp. 62-80 (2010).

8. Ahmed, M.Y.M. and Qin, N. "Numerical investigation of aeroheating characteristics of spiked blunt bodies at Mach six flight conditions", The Aeronautical Journal, 115(1168), pp. 377-386 (2011).

9. Ahmed, M.Y.M. and Qin, N. "Recent advances in the aerothermodynamics of spiked hypersonic vehicles", Progress in Aerospace Sciences, 47, pp. 425-449 (2011).

10. Gauer, M. and Paull, A. "Numerical investigation of spiked blunt nose cone at hypersonic speeds", Journal of Spacecraft and Rockets, 45(3), pp. 459-471 (2008).

11. Tahani, M., Karimi, M.S., Motlagh, A.M., and Mirmahdian, S. "Numerical investigation of drag and heat reduction in hypersonic spiked blunt bodies", Heat and Mass Transfer, 49, pp. 1369-1384 (2013).

12. Gerdroodbary, M.B. and Hosseinalipour, S.M. "Numerical simulation of hypersonic flow over highly blunted cones with spike", Acta Astronautica, 67, pp. 180-193 (2010).
13. dHumieres, G. and Stollery, J.L. "Drag reduction on a spiked body at hypersonic speed", The Aeronautical Journal, 114(1152), pp. 113-119 (2010).

14. Sebastian, J.J., James, S.E., and Suryan, A. "Computational study of hypersonic flow past spiked blunt body using RANS and DSMC method", Procedia Technology, 25, pp. 892-899 (2016).

15. Eghlima, Z. and Mansour, K. "Drag reduction for the combination of spike and counterflow jet on blunt body at high Mach number flow", Acta Astronautica, 133, pp. 103-110 (2017).

16. Qin, Q., Xu, J., and Guo, S. "Fluid-thermal analysis of aerodynamic heating over spiked blunt body configurations", Acta Astronautica, 132, pp. 230-242 (2017).

17. Yadav, R., Velidi, G., and Guven, U. "Aerothermodynamics of generic re-entry vehicle with a series of aerospikes at nose", Acta Astronautica, 96, pp. 1-10 (2014).

18. Sahoo, D., Das, S., Kumar, P., and Prasad, J.K. "Effect of spike on steady and unsteady flow over a blunt body at supersonic speed", Acta Astronautica, 128, pp. 521-533 (2016).

19. Das, S., Kumar, P., Ralh, M.K., Rao, R.K.M., and Prasad, J.K. "Drag reduction of a hemispherical body adopting spike at supersonic speed", Jr. of Aerospace Sciences and Technologies, 65(4), pp. 313-325 (2013).

\section{Biographies}

Sudip Das obtained his $\mathrm{PhD}$ in Engineering from Birla Institute of Technology, Mesra, and a post-doc from Technion, Israel Institute of Technology. He is presently working as Professor in the Department of Space Engineering and Rocketry, BIT Mesra. He has guided many students for Master's programme and is currently guiding PhD students. He has many publications in journals and conference proceedings. $\mathrm{He}$ is a member of Aeronautical Society of India, Institution of Engineers (I), Indian Society for Technical Education, and Society of Fluid Mechanics and Fluid Power. His area of research is experimental aerodynamics.

Priyank Kumar obtained his MEng and $\mathrm{PhD}$ in Engineering from Birla Institute of Technology, Mesra Ranchi. He is presently working as Assistant Professor in the Department of Space Engineering and Rocketry, BIT Mesra. He has good number of publications in journals and conference proceedings. He is a member of Institution of Engineers (I), Indian Society for Technical Education, and Society of Fluid Mechanics and Fluid Power. His area of research is fluid mechanics.

Jai Kumar Prasad obtained his $\mathrm{PhD}$ in Aerospace Engineering from Indian Institute of Technology, Madras, in 1992. Before joining Birla Institute of 
Technology, Mesra, as Professor in 2001, he worked as Scientist/Engineer and Project Manager at Vikram Sarabhai Space Centre, Thiruvananthapuram, from 1977 to 2001. He had been to DLR Gottingen, Germany, as visiting scientist and CALTECH, USA, as research fellow. Currently, he is working as Director at Deoghar Center of Birla Institute of Technology. He has guided many students for Master's programme and $\mathrm{PhD}$ students. He has a number of publications in national and international journals and conference proceedings. His areas of research are aerodynamics, jets, jet interactions, etc. 